\title{
Virtual Reality-based Vision Therapy Versus OBVAT in the Treatment of Convergence Insufficiency, Accommodative Dysfunction: A Randomized Controlled Trial
}

\author{
Shijin Li \\ Sichuan University \\ Angcang Tang \\ West China Hospital of Sichuan University \\ Bi Yang \\ West China Hospital of Sichuan University \\ Jianglan Wang \\ West China Hospital of Sichuan University \\ Longqian Liu ( $\sim$ b.q15651@hotmail.com ) \\ West China Hospital of Sichuan University
}

\section{Research Article}

Keywords: vision therapy, virtual reality, convergence insufficiency, accommodative dysfunction, binocular vision

Posted Date: October 8th, 2021

DOl: https://doi.org/10.21203/rs.3.rs-923595/v1

License: (c) This work is licensed under a Creative Commons Attribution 4.0 International License.

Read Full License 


\section{Abstract}

Background: Virtual reality is being increasingly applied in vision therapy. However, the differences in effectiveness, optimal treatment cycle, and prognosis between virtual reality-based vision therapy and traditional therapies remain unknown. The purpose of this study was to compare the effectiveness of virtual reality-based vision therapy and office-based vergence/accommodative therapy in young adults with convergence insufficiency or accommodative dysfunction.

Methods: The patients were randomly assigned to either the virtual reality-based vision therapy group or the office-based vergence/accommodative therapy group. The vision therapy lasted 12 weeks ( $1 \mathrm{~h} /$ week) in both groups. Binocular visual functions (vergence and accommodation) were measured and a subjective questionnaire-based assessment was performed at baseline and after 6 and 12 weeks of therapy.

Results: In total, 33 patients with convergence insufficiency and 30 with accommodative dysfunction completed the study. After 12 weeks of treatment for convergence insufficiency, the Convergence Insufficiency Symptom Survey score $\left(F_{2,31}=13.704, P<0.001\right)$, near point of convergence $\left(F_{2,31}=21.774\right.$, $P<0.001)$, positive fusional vergence $\left(F_{2,31}=71.766, P<0.001\right)$, and near horizontal phoria $\left(F_{2,31}=\right.$ $16.482, \mathrm{P}<0.001)$ improved significantly in both groups. Moreover, the monocular accommodative amplitude $\left(F_{2,25}=22.154, P<0.001\right)$ and monocular accommodative facility $\left(F_{2,25}=86.164, P<0.001\right)$ improved significantly in both groups after 12 weeks of treatment. A statistically significant difference was observed in monocular accommodative facility $\left(F_{1,25}=8.140, P=0.009\right)$ between the two groups, but not in other vergence and accommodative functions $(0.098<\mathrm{P}<0.687)$.

Conclusion: Virtual reality-based vision therapy significantly improved binocular vision functions and symptoms in patients with convergence insufficiency and accommodative dysfunction, thereby suggesting its effectiveness as a new optional or additional treatment for young adults with these conditions.

Trial registration: This study was registered at the Chinese Clinical Trials Registry on 16/04/2019 (identifier: ChiCTR1900022556).

\section{Introduction}

Binocular vision is important for hand-eye coordination[1], reading[2], stereopsis[3], and academic performance[4]. Patients with convergence insufficiency (CI) or accommodative dysfunction (AD) have impaired binocular vision. When patients with convergence insufficiency view a near visual target, one or both eyes tend to drift outward. They exhibit several symptoms, such as sore eyes, eye pain, headache, blurred vision, and double vision. Patients with accommodative dysfunction have difficulty focusing on targets and may experience blurred vision, headache, and fatigue associated with tasks at a near viewing distance and holding reading material close or away. The prevalence of convergence insufficiency and 
accommodative dysfunction is approximately $2.7-17.6 \%[5-9]$ and $2.3-20.2 \%[10,11]$, respectively, in the general population. Both conditions have been shown to cause asthenopia, whose prevalence has increased owing to the widespread use of electronics and prolonged near work, especially among young adults $[12,13]$.

Previous studies on children and adults have shown that vision therapy can effectively improve binocular vision functions and relieve asthenopia. The Convergence Insufficiency Treatment Trial (CITT) study group $[14,15]$ reported that office-based vergence/accommodative therapy (OBVAT) was more effective than home-based push-up therapy, computer-based vision therapy, and office-based placebo therapy for improving binocular function in patients with convergence insufficiency. Other studies[16,17] also showed that OBVAT produced significant improvements in monocular accommodative amplitude and facility. Some studies $[18,19]$ that investigated the effects of OBVAT on attention and reading in children with convergence insufficiency found that it could improve reading comprehension, reading speed, and academic behavior. The improvements in attention and reading were highly consistent with improvements in binocular functions. Therefore, vision therapy is important for patients with convergence insufficiency.

However, the implementation of OBVAT requires the guidance of a professional optometrist. Additionally, OBVAT is boring for some patients. Therefore, new treatment options must be explored.

Virtual reality (VR) has been implemented in vision therapy such as amblyopia. Studies have shown that virtual reality training improves not only amblyopia treatment compliance[20] but also the visual acuity of the amblyopic eye in adults with anisometropic amblyopia[21]. A few studies have also used virtual reality for treating convergence insufficiency and accommodative dysfunction. Yaramothu et al.[22] found that virtual reality therapy effectively improved the vergence function and relieved symptoms of convergence insufficiency. Moreover, Boon et al.[23] found that a virtual reality therapy group had higher compliance when patients with convergence insufficiency were trained using anaglyphs and a virtual reality game of snakes. Munsamy et al.[24] reported that virtual reality gaming for 25 min improved accommodative and vergence facility. These studies suggested the utility of virtual reality-based vision therapy for treating convergence insufficiency and accommodative dysfunction.

Therefore, this study aimed to compare the effectiveness of virtual reality-based vision therapy and OBVAT for young adults with convergence insufficiency and accommodative dysfunction.

\section{Methods}

\section{Study design}

In this single-center, prospective, longitudinal trial, patients with convergence insufficiency or accommodative dysfunction were randomly allocated to either the virtual reality or OBVAT group in a 1:1 ratio using a random allocation sequence generated with the Clinical Trial Management Public Platform. 
The examiner who measured the outcome measures before and after treatment will be masked to treatment assignment. Figure 1 presents the study flowchart.

\section{Patients}




\section{Table 1}

Inclusion criteria, exclusion criteria and diagnostic criteria

\section{Inclusion criteria}

Age 18-35 years

Best correct visual acuity of $20 / 25$ or better in each eye at distance (5 m) and near (40 $\mathrm{cm}$ )

Random-dot stereopsis no less than 480 seconds of arc $(40 \mathrm{~cm})$

No previous prism or near add before study enrollment

Willing to wear appropriate refractive correction for at least 2 weeks before vision therapy (spectacles are required for diopters that meet the following criteria).

Myopia $\leq-0.75 \mathrm{D}$ spherical equivalent in either eye

Hyperopia $\geq+2.00 \mathrm{D}$ spherical equivalent in either eye

Anisometropia $\geq 0.75 \mathrm{D}$ spherical equivalent

Astigmatism $\geq 1.00 \mathrm{D}$ in either eye

\section{Exclusion criteria}

$\geq 2 \triangle$ esophoria at distance

History of strabismus, amblyopia, manifest or latent nystagmus

History of vision therapy

Organic lesions of the eye

History of any ocular surgery

History of head trauma or known disease of the brain

Systemic or mental diseases, such as diabetes or anxiety, affecting accommodation, vergence, and ocular motility

Use of ocular or systemic medications containing atropine, pirenzepine, or antiepileptic in the past 3 months

\section{Diagnostic criteria}

\section{Convergence Insufficiency}

We enrolled participants with $\mathrm{Cl}$ who had a near exophoria at least $4 \triangle$ greater than distance exophoria and met at least two of the following three criteria:

1) Near point of convergence break point $\geq 6 \mathrm{~cm}$ 
2) Reduced near positive fusional vergence $₫$ break point $\leq 15 \triangle$ or failed Sheard's criterion》

3) Convergence Insufficiency Survey Score $\geq 21$ points

\section{Accommodative Dysfunctions}

We enrolled participants with AD who met at least one of the following two criteria:

1) The monocular amplitude of accommodation $\geq 2$ diopters below the minimum prediction(15-age/4)

2) Monocular accommodative infacility $\leq 6$ cycles per minute with $\pm 2.00 \mathrm{D}$ lenses

The patients were enrolled between May 2019 and October 2020 at the Department of Ophthalmology, West China Hospital of Sichuan University. The study was approved by the West China Hospital of Sichuan University Biomedical Research Ethics Committee. The study conformed to the tenets of the Declaration of Helsinki. The patients signed informed consent before any study procedures were performed. The inclusion, exclusion, and diagnostic criteria are listed in Table 1.

\section{Ocular health examination}

Slit-lamp microscopy, scanning laser ophthalmoscopy, and tonometry examinations were used to exclude patients with eye disease. Sodium fluorescein paper was used to test the tear break-up time and exclude patients with dry eye.

\section{Clinical outcome measures}

The patients received binocular vision examinations and completed subjective questionnaires (Convergence Insufficiency Symptom Survey, CISS) at baseline and after 6 and 12 weeks of therapy. The examination could not be performed during the same day as the training, but it was completed within 1 week after the last training. Binocular vision functions were tested under best-corrected refraction. The primary outcome measure of convergence insufficiency was the CISS score, and the secondary outcome measures were near point of convergence (NPC), positive fusional vergence (PFV), and near horizontal phoria. The primary outcome measures of accommodative dysfunction were monocular accommodative amplitude and facility. In this study, only the amplitude and facility of the right eye were reported because the left eye and binocular amplitude and facility had a similar tendency to those of the right eye.

\section{Treatment}

All therapies were conducted under best-corrected refraction. 


\section{Virtual reality-based vision therapy group}

Virtual reality-based vision therapy was based on the principle of dividing two similar images (targets) into both eyes. This was accomplished by dissociating the eyes with two screens (Figure 2). The screen resolution was $1920 \times 1080$ pixels, and the display refresh frequency was $120 \mathrm{~Hz}$.

\section{Virtual reality-based vision therapy for vergence}

\section{A. Stimuli}

Visual targets presented on the virtual reality screen were horizontally separated and induced vergence demands. The targets were two similar images for two eyes and included human figures, animal figures, or objects (e.g., gourds) (Figure 3A). The background was blurred to highlight the target at the center. The two images approached or moved away simultaneously to change the vergence demands, which ranged from $20^{\Delta}$ base-in to $30^{\Delta}$ base-out, and the eyes were trained for both convergence and divergence.

\section{B. Task}

The patients were asked whether they saw a figure that appeared to be floating closer than the plane of the screen (Figure 3B). The targets, which were selected by the therapist, varied in their level of complexity, thereby affecting the ability of the observer to fuse the images properly. For instance, visual targets with simple lines, larger areas, and less fine detail were easier to fuse, whereas those with complex shapes and finer detail were more difficult to fuse.

The patients first needed to fuse the two targets into one at the zero prismatic demand position. The instrument then increased the prismatic demand at 2 PD per second. To ensure that the patients received proper training during the therapy, they had to maintain a clear, fused vision. If they saw a double image and/or lost depth perception, they immediately reported it to the therapist, who would restart the vergence demand from zero.

Figure $3 \mathrm{~A}$ shows the images seen by each eye, with the two images moving simultaneously to produce a converging or diverging stimulus. Figure $3 \mathrm{~B}$ shows the complete stereo image after fusion. Figure $3 \mathrm{C}$ shows balls of different sizes and colors with optotypes appearing on the screen. In training sequences, balls appeared on the screen one by one.

\section{Training procedure}

There were 15 trials with different targets and different vergence demands. At each visit, the patients performed 4 to 5 procedures. Each training session lasted approximately 8 to $10 \mathrm{~min}$, with a short break 


\section{Virtual reality-based vision therapy for accommodation}

\section{A. Stimuli}

In the training sequences, balls of different sizes and colors appeared sequentially on the screen (Figure 3C). The optotype on the balls was the letter 'E' at different sizes, as is the case in the visual acuity chart. The background was blurred to highlight the targets. The balls were moved from far to near or vice versa by changing the binocular parallax. A ball with an optotype appeared on the screen every 1.5 to $3 \mathrm{~s}$.

\section{B. Task}

During ball movement, the patients were required to always keep the optotype clear. Usually, the task began with slower moving balls and larger targets. To ensure that the patients received proper training during the therapy, they had to maintain clear vision. If the patients saw a blurred optotype, they immediately reported it to the therapist and proceeded with the training protocol after a short break.

\section{Training procedure}

There were 8 trials with different sequences of targets and ball movement speeds. Each session lasted approximately 5 to $8 \mathrm{~min}$, with a short break between sessions.

\section{OBVAT group}

The treatment followed the CITT therapy protocol[25]. The OBVAT procedures were divided into three phases. Phases 1 and 2 included the Brock string and Barrel card tests for cross convergence and vectograms, LifeSaver cards, aperture rule, and eccentric circles for fusional vergence. Phase 3 mainly focused on jump vergence and added prism facility. For accommodative therapy, phases 1 and 2 included letter chart, lens sorting, and monocular accommodative facility. Phase 3 added binocular accommodative facility. Personalized training programs were used according to each patient's situation and training progress.

\section{Statistical analysis}

The sample size of $\mathrm{Cl}$ was calculated based on the outcome measures reported in the previous study of VR and the CITT Manual of Procedures. The mean improvements in the CISS score after 12 weeks were 16.2 and 10, respectively. In addition, the predicted standard deviation was 5.5. Power analysis (power of 
$80 \%$ and significance level of $5 \%$ ) indicated that 14 participants were needed for each group, and we estimated that approximately $20 \%$ of participants might withdraw. Therefore, a total of 18 participants with $\mathrm{Cl}$ were randomly assigned to each treatment group.

All data analyses were performed using IBM SPSS Statistics for Windows, Version 23.0 (IBM Corp., Armonk, NY). A chi-square test was used to compare sex differences between the groups. An independent t-test was used to compare the baseline findings between the groups. Repeated-measures analysis of variance was used to determine any significant effect of visit and group on binocular functions and symptoms (within-subjects factor: duration of treatment, between-subjects factor: treatment group). The Pearson chi-square test was used to compare the proportion of each indicator reaching normal in the two groups. P values $<0.05$ were considered statistically significant.

\section{Results}

This study enrolled 36 patients with convergence insufficiency and 34 with accommodative dysfunction; 7 patients ( 3 with convergence insufficiency and 4 with accommodative dysfunction) were lost to followup. Finally, 33 patients with convergence insufficiency and 30 with accommodative dysfunction completed the study. The demographics and baseline data of the patients are shown in Table 2. No significant intergroup differences were observed in age, refraction, horizontal phoria at distance or near, NPC, gradient or calculated ratio of accommodative convergence to accommodation, PFV, CISS scores, negative relative accommodation, positive relative accommodation, or binocular cross-cylinder. 
Table 2

Study population demographics and clinical measures at baseline

\begin{tabular}{|c|c|c|c|}
\hline & $\begin{array}{l}\text { Virtual reality-based } \\
\text { vision therapy, } \\
\text { mean } \pm \text { SD }\end{array}$ & $\begin{array}{l}\text { Office-based } \\
\text { vergence/accommodative therapy, } \\
\text { mean } \pm \text { SD }\end{array}$ & $\begin{array}{l}P \\
\text { value }\end{array}$ \\
\hline Sex, M: F & 7:17 & $7: 18$ & 0.830 \\
\hline Age (year) & $23.21 \pm 1.80$ & $23.08 \pm 1.91$ & 0.809 \\
\hline $\begin{array}{l}\text { Right eye spherical } \\
\text { equivalent, (D) }\end{array}$ & $-4.12 \pm 2.04$ & $-3.94 \pm 2.14$ & 0.778 \\
\hline $\begin{array}{l}\text { Left eye spherical } \\
\text { equivalent, (D) }\end{array}$ & $-3.89 \pm 2.01$ & $-3.47 \pm 2.49$ & 0.530 \\
\hline $\begin{array}{l}\text { Phoria at distance (cover } \\
\text { test) }(\triangle)\end{array}$ & $-3.75 \pm 3.62$ & $-2.88 \pm 2.76$ & 0.443 \\
\hline $\begin{array}{l}\text { Phoria at near (cover test) } \\
(\triangle)\end{array}$ & $-16.31 \pm 3.99$ & $-15.35 \pm 2.83$ & 0.430 \\
\hline NPC break (cm) & $6.60 \pm 3.63$ & $6.00 \pm 2.95$ & 0.763 \\
\hline Gradient $\mathrm{AC} / \mathrm{A}$ ratio & $2.44 \pm 2.21$ & $2.68 \pm 2.29$ & 0.979 \\
\hline Calculated $\mathrm{AC} / \mathrm{A}$ ratio & $1.59 \pm 1.40$ & $1.61 \pm 1.16$ & 0.609 \\
\hline PFV break at near, $(\triangle)$ & $22.53 \pm 6.15$ & $23.06 \pm 5.18$ & 0.828 \\
\hline PFV recovery at near, $(\triangle)$ & $9.03 \pm 8.85$ & $9.88 \pm 7.31$ & 0.765 \\
\hline CISS score & $27.69 \pm 8.46$ & $25.18 \pm 9.40$ & 0.427 \\
\hline NRA, (D) & $2.38 \pm 0.40$ & $2.47 \pm 0.25$ & 0.583 \\
\hline PRA, (D) & $-2.90 \pm 1.75$ & $-2.59 \pm 0.65$ & 0.644 \\
\hline BCC, (D) & $0.06 \pm 1.06$ & $0.34 \pm 1.02$ & 0.596 \\
\hline $\begin{array}{l}\text { Monocular } \\
\text { accommodative } \\
\text { amplitude, (D) }\end{array}$ & $10.94 \pm 3.76$ & $10.41 \pm 2.66$ & 0.750 \\
\hline $\begin{array}{l}\text { Monocular } \\
\text { accommodative facility, } \\
(\mathrm{cpm})\end{array}$ & $6.31 \pm 2.56$ & $4.94 \pm 4.44$ & 0.461 \\
\hline \multicolumn{4}{|c|}{$\begin{array}{l}\text { NPC: near point of convergence, AC/A: accommodative convergence to accommodation, PFV: } \\
\text { positive fusional vergence, CISS: Convergence Insufficiency Symptoms Survey, NRA: negative relative } \\
\text { accommodation, PRA: positive relative accommodative, BCC: binocular cross-cyclinder, D: diopters, } \triangle \text { : } \\
\text { prism diopters, cpm: cycles per minute. }\end{array}$} \\
\hline
\end{tabular}

Changes in symptoms and vergence function 
The clinical measures of patients with convergence insufficiency at baseline and after 6 and 12 weeks are presented in Table 3. 
Table 3

Changes in outcome measures of patients with convergence insufficiency by treatment group

\begin{tabular}{|c|c|c|c|c|}
\hline CISS score & Baseline & 6 weeks & 12 weeks & Total change \\
\hline VR group & $27.69 \pm 8.50$ & $22.63 \pm 9.88$ & $21.75 \pm 9.13$ & $-5.94 \pm 3.11$ \\
\hline OBVAT group & $25.18 \pm 9.40$ & $18.82 \pm 8.15$ & $16.47 \pm 8.02$ & $-8.71 \pm 2.99$ \\
\hline Effect size & group & group*time & time & \\
\hline F value & 2.298 & 0.424 & 13.074 & \\
\hline$P$ value & 0.140 & 0.656 & $<0.001$ & \\
\hline NPC break (cm) & Baseline & 6 weeks & 12 weeks & Total change \\
\hline VR group & $6.59 \pm 3.63$ & $5.06 \pm 2.73$ & $4.62 \pm 2.38$ & $-1.97 \pm 1.09$ \\
\hline OBVAT group & $6.00 \pm 2.95$ & $3.91 \pm 1.99$ & $3.82 \pm 1.41$ & $-2.18 \pm 0.80$ \\
\hline Effect size & group & group*time & time & \\
\hline$F$ value & 1.084 & 0.478 & 13.290 & \\
\hline$P$ value & 0.306 & 0.624 & $<0.001$ & \\
\hline PFV blur or break at near $(\Delta)$ & Baseline & 6 weeks & 12 weeks & Total change \\
\hline VR group & $22.63 \pm 6.15$ & $32.97 \pm 7.98$ & $34.47 \pm 5.20$ & $11.84 \pm 2.01$ \\
\hline OBVAT group & $23.06 \pm 5.18$ & $34.68 \pm 5.98$ & $35.47 \pm 4.64$ & $12.41 \pm 1.69$ \\
\hline Effect size & group & group*time & time & \\
\hline F value & 0.421 & 0.008 & 61.222 & \\
\hline$P$ value & 0.521 & 0.888 & $<0.001$ & \\
\hline Near horizontal phoria $(\Delta)$ & Baseline & 6 weeks & 12 weeks & Total change \\
\hline VR group & $-16.31 \pm 3.99$ & $-13.94 \pm 4.60$ & $-13.13 \pm 5.15$ & $3.19 \pm 1.63$ \\
\hline OBVAT group & $-15.35 \pm 2.83$ & $-11.53 \pm 5.62$ & $-9.94 \pm 6.33$ & $5.41 \pm 1.68$ \\
\hline Effect size & group & group*time & time & \\
\hline F value & 2.261 & 1.067 & 16.482 & \\
\hline$P$ value & 0.143 & 0.350 & $<0.001$ & \\
\hline
\end{tabular}

The repeated-measures analysis of variance showed a significant effect of visit on the CISS score $\left(\mathrm{F}_{2,31}=\right.$ $13.704, P<0.001)$, NPC break $\left(F_{2,31}=21.774, P<0.001\right)$, PFV break $\left(F_{2,31}=71.766, P<0.001\right)$, and near 
horizontal phoria $\left(F_{2,31}=16.482, P<0.001\right)$. To control for the baseline differences between the groups, the measures at 6 and 12 weeks were normalized to the baseline values (ex. 12-week data - baseline data), but the repeated-measures analysis of variance also showed no statistically significant effect of group on the measures of the CISS score $\left(F_{1,31}=0.437, P=0.514\right)$, NPC break $\left(F_{1,31}=0.258, P=0.615\right)$, $\operatorname{PFV}$ break $\left(F_{1,31}=0.166, P=0.687\right)$, and near horizontal phoria $\left(F_{1,31}=1.484, P=0.232\right)$. Moreover, the post hoc comparison showed no statistically significant effect of 6 and 12 weeks of therapy in either group $(0.237<\mathrm{P}<0.675)$.

After 12 weeks of therapy, $81.3 \%$ of patients in the virtual reality group and $88.2 \%$ in the OBVAT group had a NPC less than $6 \mathrm{~cm}$ and PFV greater than or equal to twice that of near phoria. The difference in the proportion of NPC and PFV reaching normal between the two groups was not statistically significant $\left(\mathrm{c}^{2}=\right.$ $0.313, \mathrm{P}=0.576$ ). Only 9 patients in the virtual reality group and 11 in the OBVAT group had Convergence Insufficiency Symptom Survey scores smaller than 21.

\section{Changes in accommodative function}

The clinical measures of patients with accommodative dysfunction at baseline and after 6 and 12 weeks are presented in Table 4. The repeated-measures analysis of variance showed a significant effect of visits on monocular accommodative amplitude $\left(\mathrm{F}_{2,25}=22.154, \mathrm{P}<0.001\right)$ and facility $\left(\mathrm{F}_{2,25}=86.164, \mathrm{P}<\right.$ 0.001). To control for baseline differences between the groups, measures at 6 and 12 weeks were normalized to baseline values. The repeated-measures analysis of variance also showed no statistically significant effect of group on the measures of monocular accommodative amplitude $\left(F_{1,25}=2.958, P=\right.$ 0.098), but showed a statistically significant difference in monocular accommodative facility between the two groups $\left(F_{1,25}=8.140, P=0.009\right)$. Moreover, the post hoc comparison showed a statistically significant difference in monocular accommodative amplitude $(P=0.002)$ and facility $(P<0.001)$ between 6 and 12 weeks of therapy. 
Table 4

Changes in outcome measures of patients with accommodative dysfunctions by treatment group

\begin{tabular}{|lllll|}
\hline $\begin{array}{l}\text { Monocular accommodative amplitude, } \\
\text { (D) }\end{array}$ & Baseline & $\mathbf{6}$ weeks & $\mathbf{1 2}$ weeks & $\begin{array}{l}\text { Total } \\
\text { change }\end{array}$ \\
\hline VR group & $10.94 \pm 3.76$ & $12.40 \pm 3.41$ & $13.86 \pm 3.35$ & $2.91 \pm 1.78$ \\
\hline OBVAT group & $10.41 \pm 2.66$ & $12.64 \pm 3.28$ & $15.15 \pm 2.79$ & $4.73 \pm 1.36$ \\
\hline Effect size & group & group*time & time & \\
\hline F value & 0.035 & 1.324 & 27.202 & \\
\hline P value & 0.853 & 0.274 & $<0.001$ & \\
\hline $\begin{array}{l}\text { Monocular accommodative facility, } \\
\text { (cpm) }\end{array}$ & Baseline & 6 weeks & 12 weeks & $\begin{array}{l}\text { Total } \\
\text { change }\end{array}$ \\
\hline VR group & $6.31 \pm 2.56$ & $13.38 \pm 5.62$ & $16.81 \pm 7.34$ & $10.50 \pm 2.75$ \\
\hline OBVAT group & $4.94 \pm 4.44$ & $12.75 \pm 4.74$ & $17.25 \pm 2.58$ & $12.31 \pm 1.82$ \\
\hline Effect size & group & group*time & time & \\
\hline F value & 0.631 & 1.636 & 86.164 & \\
\hline P value & 0.434 & 0.213 & $<0.001$ & \\
\hline D: diopters; cpm: cycles per minute & & & & \\
\hline
\end{tabular}

After 12 weeks of therapy, one patient in the virtual reality group and one in the OBVAT group still had monocular accommodative amplitudes less than the minimum prediction (15-age/4) after 12 weeks of therapy. While 5 patients in the virtual reality group still had monocular accommodative facility less than $12 \mathrm{cpm}$, none in the OBVAT group did. The Pearson chi-square with Fisher's exact test showed a statistically significant intergroup difference in effectiveness in reaching the normal monocular accommodative facility $(P=0.042)$.

\section{Discussion}

In this study, the OBVAT group was chosen as the control group because OBVAT is the most effective therapy currently known for improving binocular vision functions and symptoms in patients with convergence insufficiency or accommodative dysfunction $[14,16]$. Previous studies[22, 23] have shown that virtual reality-based vision therapy is promising, and the results of the present study suggest that virtual reality training can be an effective method for patients with convergence insufficiency and accommodative dysfunction.

\section{Changes in symptoms and vergence function in patients with convergence insufficiency}


After 12 weeks of treatment, the CISS score, NPC, PFV, and near horizontal phoria improved significantly in both the virtual reality and OBVAT groups. The proportion of achieving normal NPC and PFV in the virtual reality and OBVAT groups was $81.3 \%$ and $88.2 \%$, respectively. The difference between the two groups was not significant. Therefore, virtual reality-based vision therapy can be as effective as OBVAT in patients with convergence insufficiency.

In this study, the mean improvements in positive fusional vergence were 11.8 and $13.5^{\triangle}$ base-out in the virtual reality and OBVAT groups, respectively, and these were similar to the values reported in two other studies $[22,23]$ that used virtual reality therapy for adults with convergence insufficiency (3.9 and $14.7^{\triangle}$ base-out, respectively). However, the mean improvement was less than that reported in previous adult studies $[15,26,27]$ using OBVAT for vision therapy. One reason for this could be the difference in refractive errors. The patients in this study had a higher degree of myopia (mean refractive error of the right eye: $-4.02 \mathrm{D}$ ). In the studies by Singh et al.[27] and Alvarez et al.[26], the proportion of patients with myopia was only approximately $25 \%$. In an adult study of CITT[15], the mean refractive error of the right eye was -0.92 D. Patients with a higher degree of myopia might exhibit a higher level of near PFV and closer NPC than those with a lower degree of myopia[28]. Therefore, the baseline values in the present study were higher than those in previous studies, and these better baseline values potentially resulted in relatively less improvement. The second reason could be that neither group received home reinforcement therapy. Previous studies[14, 29] have found better results in groups receiving home reinforcement therapy.

Similarly, the mean improvement in the NPC was 2.08 and $2.18 \mathrm{~cm}$ in the virtual reality and OBVAT groups, respectively, which was similar to the $2.4 \mathrm{~cm}$ improvement reported in a previous study[23] that also used virtual reality therapy for adults with convergence insufficiency. However, the mean improvement in near point of convergence was less than that in previous adult studies[15,26,27] using OBVAT. This could also be attributed to the better baseline values and the absence of home reinforcement therapy in the present study groups.

However, the mean improvements in the CISS score in the present study were 5.93 and 8.71 points for the virtual reality and OBVAT groups, respectively. These values were less than those reported in a previous adult study of CITT[15]. Moreover, only 9 patients in the virtual reality group and 11 in the OBVAT group had CISS scores smaller than 21. The main reason for this could be that even during vision therapy, some patients still spent much time in near work using a computer or mobile phone. This suggests the need for changes in life and work habits to achieve improvements in symptoms. Moreover, the CISS was developed 20 years ago, and its validity may have decreased because the widespread use of electronic devices and long durations of near work have become important causative factors for asthenopia[3032]. This also suggested that in future studies, life and work habits should be quantified and included as measurement factors to evaluate the effectiveness of vision therapy.

To summarize, virtual reality-based vision therapy can offer benefits to patients with convergence insufficiency. Targeted stimuli in virtual reality allowed for the presentation of hierarchical and repetitive 
stimuli. Meanwhile, stimuli, such as vergence demands and binocular disparity, were modulated during vision therapy.

\section{Changes in accommodation in patients with accommodative dysfunction}

After 12 weeks of treatment, monocular accommodative amplitude and facility improved significantly in the virtual reality and OBVAT groups. The mean improvement and proportion of achieving normal monocular accommodative facility were statistically significant in the two groups, but those for monocular accommodative amplitude were not significant. Therefore, virtual reality-based vision therapy can be effective for patients with accommodative dysfunction. Nevertheless, its effect on improving accommodative facility was lower than that of OBVAT.

The mean improvements in monocular accommodative facility $(9.8$ and $12.5 \mathrm{cpm}$ in the virtual reality and OBVAT groups, respectively) observed in the present study were consistent with the improvements (9.4 and $10.9 \mathrm{cpm}$ ) reported in previous studies[16, 33] on children. The present study also found that monocular accommodative facility was better at 12 weeks than at 6 weeks of therapy, and the results were similar to those of previous studies[16,34]. Although monocular accommodative facility in the virtual reality group improved significantly, the effect was still lower than that in the OBVAT group. The reason could be the different training principles of accommodative function in the virtual reality and OBVAT groups. Virtual reality-based vision therapy changed binocular disparity and increased fusional accommodation, in which the accommodative plane was fixed. OBVAT used lenses with different degrees and reading materials at different distances, which produced a greater accommodative stimulus.

The mean improvements in monocular accommodative amplitude in the virtual reality and OBVAT groups were less than those reported in previous studies on children[16]. In addition to the larger baseline values in this study, age was an important factor affecting the improvement in accommodative amplitude. Moreover, improvements in accommodative function may require a longer duration than improvements in convergence insufficiency. Therefore, improving the effectiveness of virtual reality-based vision therapy for accommodative dysfunction needs further investigation.

\section{Limitations}

This study has several limitations. First, this study did not assess other factors that might affect fatigue symptoms, such as the occupational background and near work habits of the included patients. Second, no placebo control group was set in this study, and repeated measurements could also have induced training effects. Finally, a larger sample size and longer follow-up time may be needed in future research to confirm these findings.

\section{Conclusion}

Virtual reality-based vision therapy significantly improved binocular functions and symptoms in patients with convergence insufficiency and accommodative dysfunction. It may be a new optional or additional 
treatment for young adults with convergence insufficiency and accommodative dysfunction. Moreover, virtual reality-based vision therapy still needs to be optimized, diversified and personalized to improve the training effectiveness and shorten the response time.

\section{Abbreviations}

Cl: convergence insufficiency

AD: accommodative dysfunction

CITT: Convergence Insufficiency Treatment Trial

OBVAT: Office-based vergence/accommodative therapy

VR: virtual reality

CISS: Convergence Insufficiency Symptom Survey

NPC: near point of convergence

PFV: positive fusional vergence

\section{Declarations}

\section{Ethics approval and consent to participate}

The study was approved by the West China Hospital of Sichuan University Biomedical Research Ethics Committee [No.2019 review (184)]. The study conformed to the tenets of the Declaration of Helsinki. The patients signed informed consent before any study procedures were performed.

\section{Consent for publication}

Not applicable.

\section{Availability of data and materials}

Data sharing is not applicable to this article as no datasets were generated or analyzed during the current study.

\section{Competing interest}


The authors report no conflicts of interest and have no proprietary interest in any of the materials mentioned in this article.

\section{Funding}

The National Nature Science Foundation of China (grant: 82070996).

\section{Authors Contributions}

SL, AT and LL conceived and planned this study and take full responsibility for the paper. SL, AT, BY, JW and LL contributed to the analysis and interpretation of the clinical data. SL took the lead in writing the manuscript. AT contributed to revision and editing of the paper. LL supervised this study. SL and AT contributed equally to this work and should be considered as equal first authors. All authors read and approved the final manuscript.

\section{Acknowledgements}

This study was supported by a Collaborative Research Agreement between West China Hospital, Sichuan University, Zhengzhou Chengyoucheng Electronic Technology Co., Ltd (grant number: HX-H1906117)

\section{References}

1. Gonzalez DA, Niechwiej-Szwedo E: The effects of monocular viewing on hand-eye coordination during sequential grasping and placing movements. Vision Res 2016, 128:30-38.

2. Palomo-Alvarez C, MC P: Binocular function in school children with reading difficulties. Graefes Arch Clin Exp Ophthalmol 2010, 248(6):885-892.

3. Levi DM, Knill DC, Bavelier D: Stereopsis and amblyopia: A mini-review. Vision Res 2015, 114:17-30.

4. Wood JM, Black AA, Hopkins S, White SLJ: Vision and academic performance in primary school children. Ophthalmic Physiol Opt 2018, 38(5):516-524.

5. Ma M, Long W, She Z, Li W, Chen X, Xie L, Scheiman M, Liu Y, Chen X: Convergence insufficiency in Chinese high school students. Clin Exp Optom 2019, 102(2):166-171.

6. Davis A, Harvey E, Twelker J, miller J, Leonard-Green T, I. C: Convergence insufficiency, accommodative insufficiency, visual symptoms, and astigmatism in Tohono O'odham students. $J$ Ophthalmol 2016, 2016:6963976.

7. Atowa UC, Wajuihian SO, Hansraj R: Vergence profile and prevalance of non-strabismic vergence anomalies among school children in Abia State, Nigeria. Ophthalmic Epidemiol 2019, 26(2):121131. 
8. Wajuihian SO, Hansraj R: Vergence anomalies in a sample of high school students in South Africa. $J$ Optom 2016, 9(4):246-257.

9. Hussaindeen JR, Rakshit A, Singh NK, George R, Swaminathan M, Kapur S, Scheiman M, Ramani KK: Prevalence of non-strabismic anomalies of binocular vision in Tamil Nadu: report 2 of BAND study. Clin Exp Optom 2017, 100(6):642-648.

10. Wajuihian SO, Hansraj R: Accommodative anomalies in a sample of black high school students in South Africa. Ophthalmic Epidemio/ 2016, 23(5):316-323.

11. Garcia-Munoz A, Carbonell-Bonete S, Canto-Cerdan M, Cacho-Martinez P: Accommodative and binocular dysfunctions: prevalence in a randomised sample of university students. Clin Exp Optom 2016, 99:313-321.

12. Agarwal S, Goel D, Sharma A: Evaluation of the factors which contribute to the ocular complaints in computer users. J Clin Diagn Res 2013, 7(2):331-335.

13. Logaraj M, Madhupriya V, Hegde S: Computer vision syndrome and associated factors among medical and engineering students in chennai. Ann Med Health Sci Res 2014, 4(2):179-185.

14. CITT Study Group: Randomized clinical trial of treatments for convergence insufficiency in children. Arch Ophthalmol 2008, 123(10):14-24.

15. Scheiman M, Mitchell G, Cotter S, Kulp M, Cooper J, Rouse M, Borsting E: A randomized clinical trial of vision therapy/orthoptics versus pencil pushups for the treatment of convergence insufficiency in young adults. Optom Vis Sci 2005, 82(7):583-595.

16. Scheiman M, Cotter S, Kulp MT, Mitchell GL, Cooper J, Gallaway M, Hopkins KB, Bartuccio M, Chung I, Convergence Insufficiency Treatment Trial Study G: Treatment of accommodative dysfunction in children: results from a randomized clinical trial. Optom Vis Sci 2011, 88(11):1343-1352.

17. Chen AM, Roberts TL, Cotter SA, Kulp MT, Sinnott LT, Borsting EJ, Tea YC, Jones-Jordan LA, Hertle R, Mitchell GL et al: Effectiveness of vergence/accommodative therapy for accommodative dysfunction in children with convergence insufficiency. Ophthalmic Physiol Opt 2021, 41(1):21-32.

18. Scheiman M, Chase C, Borsting E, Mitchell G, Cotter S: Effect of treatment of symptomatic convergence insufficiency on reading in children: a pilot study. Clin Exp Optom 2018, 101(4):585593.

19. Borsting E, Mitchell GL, Kulp MT, Scheiman M, Amster DM, Cotter S, Coulter RA, Fecho G, Gallaway MF, Granet $D$ et al: Improvement in academic behaviors after successful treatment of convergence insufficiency. Optom Vis Sci 2012, 89(1):12-18.

20. Eastgate R, Griffiths G, Waddingham P, Moody A, Butler T, Cobb S, Comaish I, Haworth SM, Gregson RM: Modified virtual reality technology for treatment of amblyopia. Eye 2006, 20(3):370-374.

21. Halička J SE, Krasňanský M, Kapitánová K, Fedorová M, Žiak P: Visual training in virtual reality in adult patients with anisometric amblyopia.. Cesk Slov Oftalmol 2020, 76(1):24-28.

22. Yaramothu C, Vito d'Antonio- Bertagnolli J, Santos EM, Crincoli P, Rajah J, M. S, Alvarez T: Virtual eye rotation vision exercises (VERVE): a virtual reality vision therapy platform with eye tracking. Brain Stimulation 2019, 12(2):e107-e108. 
23. Boon MY, Asper LJ, Chik P, Alagiah P, Ryan M: Treatment and compliance with virtual reality and anaglyph-based training programs for convergence insufficiency. Clin Exp Optom 2020, 103(6):870876.

24. Munsamy AJ, Paruk H, Gopichunder B, Luggya A, Khulu S: The effect of gaming on accommodative and vergence facilities after exposure to virtual reality head-mounted display. J Optom 2020, 13(3):163-170.

25. CITT Manual of Procedures. Chapter 8: Office-Based Vision Therapy (VT/Orthoptics). Othe Ohio University College of Optometry. http://optometry.osu.edu/CITT-manual-procedures. Accessed 20 May 2019.

26. Alvarez TL, Vicci VR, Alkan Y, Kim EH, Gohel S, Barrett AM, Chiaravalloti N, Biswal BB: Vision therapy in adults with convergence insufficiency: clinical and functional magnetic resonance imaging measures. Optom Vis Sci 2010, 87(12):E985-1002.

27. Singh A, Saxena V, Yadav S, Agrawal A, Ramawat A, Samanta R, Panyala R, B. K: Comparison of home-based pencil push-up therapy and office-based orthoptic therapy in symptomatic patients of convergence insufficiency: a randomized controlled trial. Int Ophthalmo/ 2021, Epub ahead of print. .

28. Ma MM, Yeo ACH, Scheiman M, Chen X: Vergence and accommodative dysfunctions in emmetropic and myopic Chinese young adults. J Ophthalmol 2019, 2019:5904903.

29. Aletaha M, Daneshvar F, Mosallaei M, Bagheri A, Khalili MR: Comparison of three vision therapy approaches for convergence insufficiency. J Ophthalmic Vis Res 2018, 13(3):307-314.

30. Golebiowski B, Long J, Harrison K, Lee A, Chidi-Egboka N, Asper L: Smartphone use and effects on tear film, blinking and binocular vision. Curr Eye Res 2019, 45(4):428-434.

31. Jaiswal S, Asper L, Long J, Lee A, Harrison K, Golebiowski B: Ocular and visual discomfort associated with smartphones, tablets and computers: what we do and do not know. Clin Exp Optom 2019, 102(5):463-477.

32. Long J, Cheung R, Duong S, Paynter R, Asper L: Viewing distance and eyestrain symptoms with prolonged viewing of smartphones. Clin Exp Optom 2017, 100:133-137.

33. Ma MM, Scheiman M, Su C, Chen X: Effect of vision therapy on accommodation in myopic Chinese children. J Ophthalmol 2016, 2016:1202469.

34. Sterner B, Abrahamsson M, Sjöström A: Accommodative facility training with a long term follow up in a sample of school aged children showing accommodative dysfunction. Doc Ophthalmol 1999, 99:93-101.

\section{Figures}




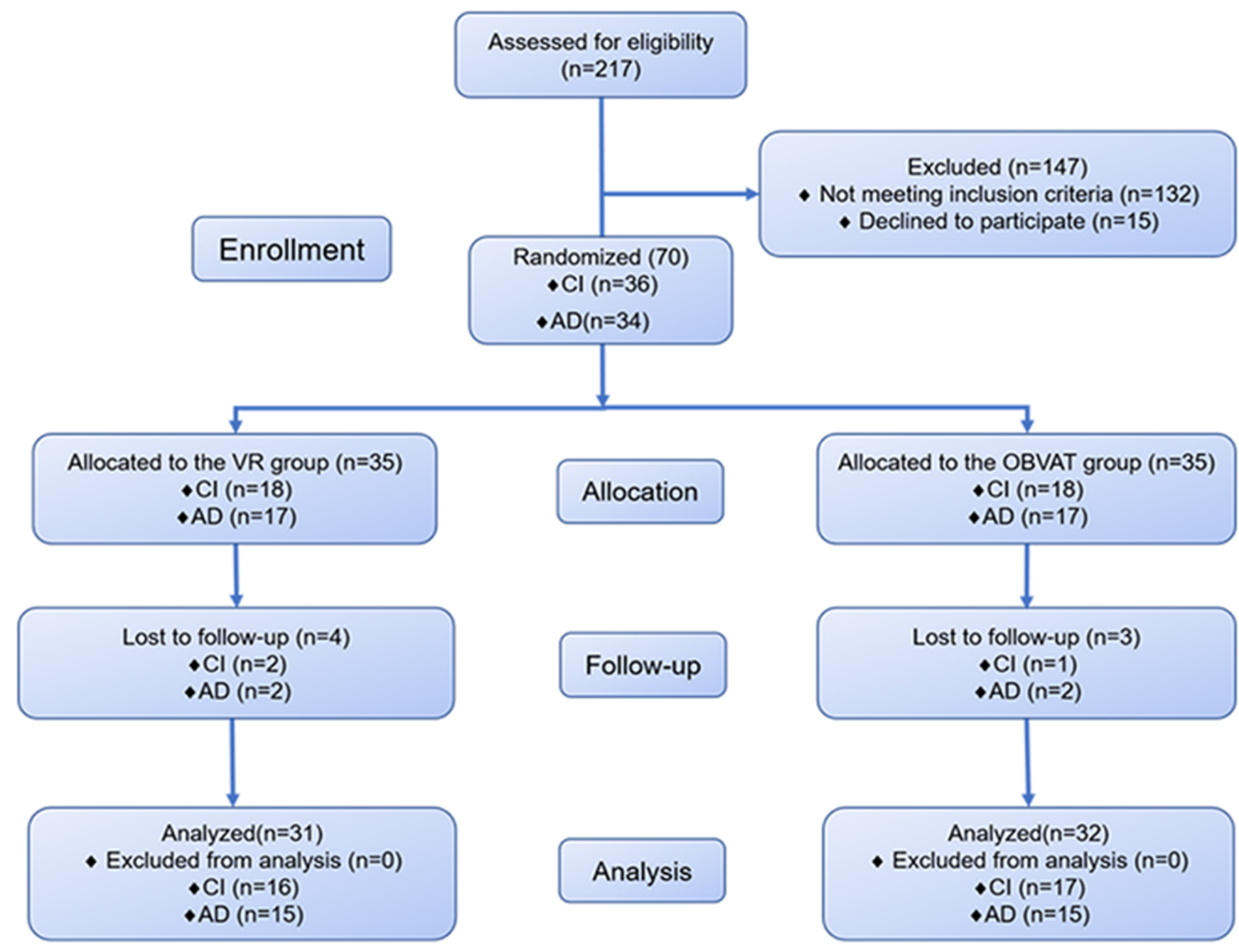

Figure 1

Flowchart showing study completion for each group. Cl: convergence insufficiency; AD: accommodative dysfunction; VR: virtual reality; OBVAT: office-based vergence/accommodative therapy 


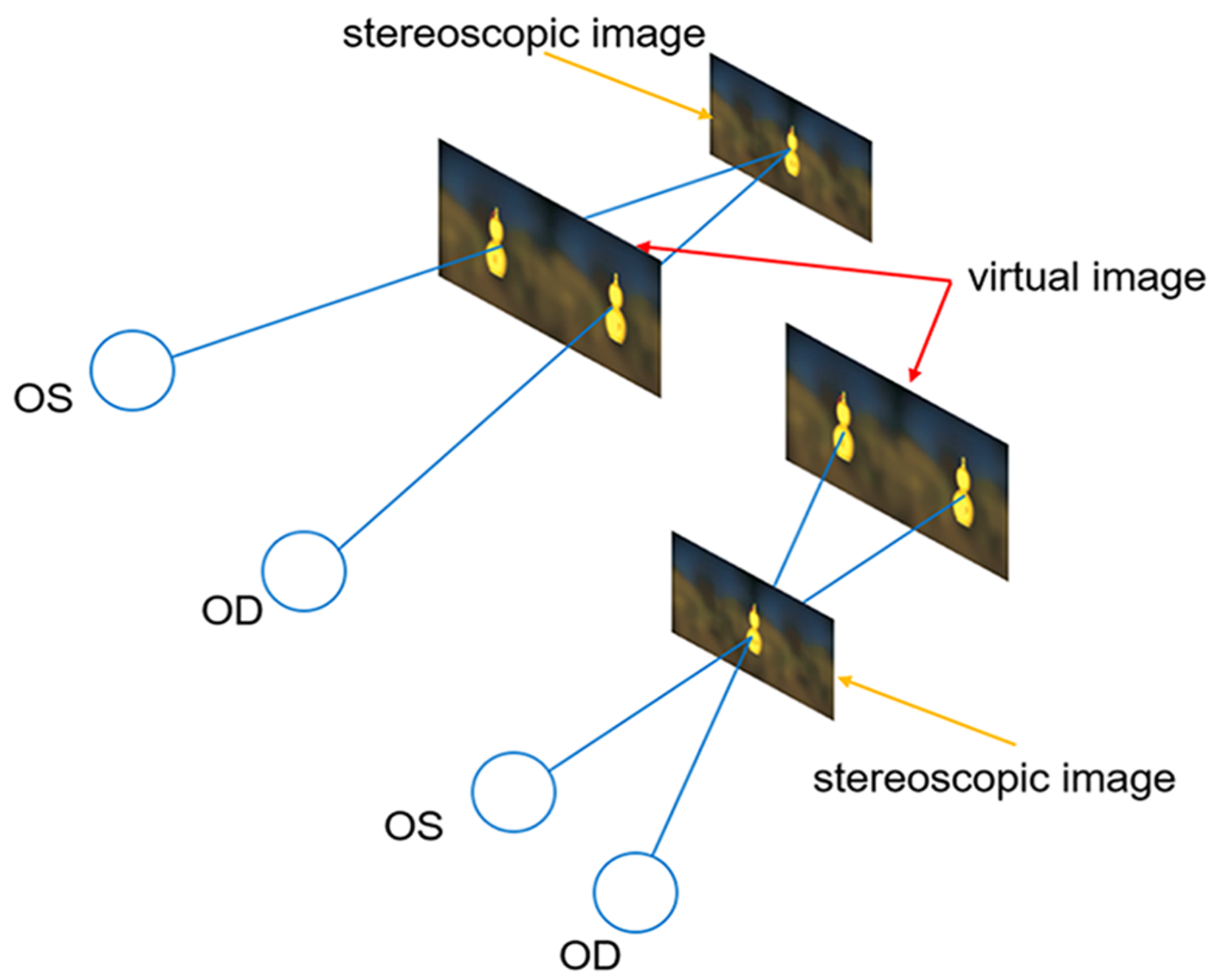

Figure 2

A simplified schematic representation of the VR imaging principles.
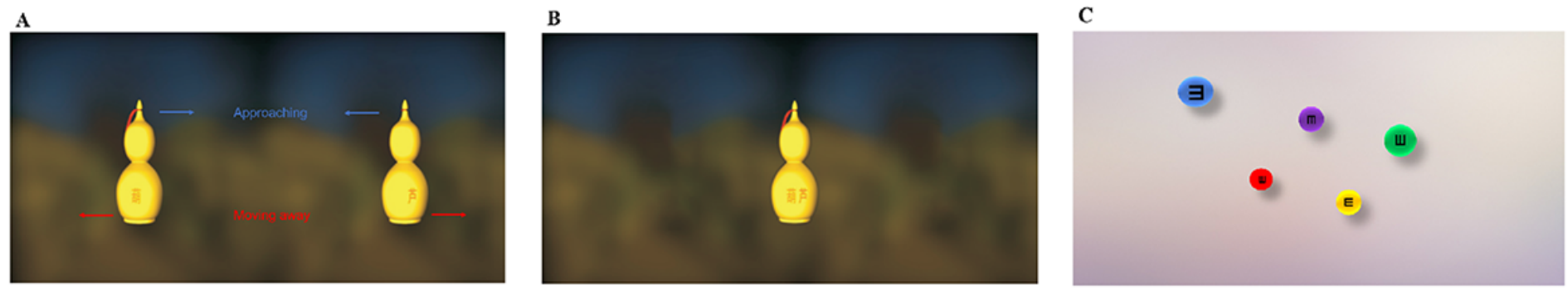

Figure 3

The schematic diagram of VR therapy. 